\title{
Inter-eye comparison of retinal oximetry and vessel caliber between eyes with asymmetrical glaucoma severity in different glaucoma subtypes
}

This article was published in the following Dove Press journal:

Clinical Ophthalmology

19 July 2016

Number of times this article has been viewed

\author{
Clarissa Shu Ming Cheng ${ }^{1,2}$ \\ Yi Fang Lee ${ }^{2}$ \\ Charles Ong ${ }^{3}$ \\ Zhu Li Yap ${ }^{2}$ \\ Andrew Tsai ${ }^{2}$ \\ Aditi Mohla ${ }^{2}$ \\ Monisha E Nongpiur ${ }^{4}$ \\ Tin Aung ${ }^{2,4}$ \\ Shamira A Perera ${ }^{2,4}$ \\ 'Department of Ophthalmology, Tan \\ Tock Seng Hospital, ${ }^{2}$ Department of \\ Ophthalmology, Singapore National \\ Eye Centre, ${ }^{3}$ Yong Loo Lin School \\ of Medicine, National University of \\ Singapore, ${ }^{4}$ Singapore Eye Research \\ Institute, Singapore National Eye \\ Centre, Singapore
}

Background: To compare retinal vessel oxygenation and vessel caliber in primary angle-closure glaucoma (PACG), primary open-angle glaucoma (POAG), normal-tension glaucoma (NTG), and normal controls, as well as between eyes of asymmetrical glaucoma severity.

Methods: This was a prospective, cross-sectional study. The 159 subjects (PACG, $n=39$; POAG, $\mathrm{n}=41$; NTG, $\mathrm{n}=41$; normal controls, $\mathrm{n}=38$ ) underwent retinal oxygen saturation measurements using the Oxymap T1 Retinal Oximeter, optical coherence tomography, and Humphrey visual field testing. Retinal oxygen saturation and vessel diameter were compared between the glaucoma groups and normal controls, as well as between eyes of asymmetrical glaucoma severity. Kruskal-Wallis test was performed for comparison among different subtypes of glaucoma. Wilcoxon signed-rank test was used to compare the inter-eye differences.

Results: Compared to normal controls, arteriolar oxygen saturation was increased in PACG eyes $(P=0.048)$ but not in POAG or NTG eyes. There were no significant differences in oxygen saturation in venules or arteriovenous (AV) difference in all three glaucoma groups. Venular diameter was significantly reduced in all glaucoma groups compared to normal controls $(P<0.001)$, but no such change was observed in arteriolar diameter $(P=0.10)$. When comparing between eyes of asymmetrical glaucoma severity, arteriolar oxygen saturation $(P=0.03)$ and AV difference $(P=0.04)$ were significantly higher, while arteriolar diameter was significantly lower $(P=0.001)$ in the worse eye in PACG group. There were no significant differences in oximetric parameters or vessel calibers between the worse and the better eyes in POAG and NTG groups.

Conclusion: Eyes with PACG showed increased arteriolar oxygen saturation and increased AV difference. This was not observed in POAG and NTG eyes. Arteriolar diameter in PACG and venular diameter in all three glaucoma groups were reduced. The difference observed in PACG eyes may be due to an increased metabolic demand in the disease process compared to open-angle glaucoma.

Keywords: glaucoma, oximetry, retinal vessels, imaging

\section{Introduction}

The pathogenesis of glaucomatous optic neuropathy $(\mathrm{GON})$ is postulated to be a combination of mechanical and vascular factors. ${ }^{1}$ The mechanical theory of barotrauma to the nerve and subsequent cell death has been thought to be the predominant cause in highpressure glaucomas such as primary open-angle glaucoma (POAG) and primary angleclosure glaucoma (PACG). ${ }^{2}$ However, differences in visual field (VF) defects between $P O A G$ and $\mathrm{PACG}^{3}$ and progression of $\mathrm{GON}$ despite normalization of intraocular pressure (IOP) suggest coexistence of pressure independent factors. The vascular theory proposes hypoperfusion and subsequent ischemia, hypoxia, inflammation, and tissue damage in
Correspondence: Shamira A Perera Singapore National Eye Centre, II Third Hospital Avenue, Singapore 16875I

Tel +6563224584

Fax +6563231903

Email shamira.perera@snec.com.sg 
the GON pathogenesis. ${ }^{4-6}$ This has been particularly evident in normal-tension glaucoma (NTG), ${ }^{7,8}$ and there is mounting evidence implicating vascular compromise in POAG. ${ }^{9-14}$

Retinal oximetry is a new noninvasive imaging technology that measures oxygen saturation levels in retinal vessels, acting as a surrogate marker for the metabolic demands of the eye. To date, six papers have been published looking at the influence of glaucoma on retinal vessel oxygen saturation. Michelson and Scibor compared POAG and NTG with normal controls and found significant correlation between the neuroretinal rim area and attenuated arteriovenous (AV) differences, suggesting reduced metabolism reflected tissue loss. They postulated that the high IOPs seen in POAG impede oxygen extraction. ${ }^{10}$ Ito et al correlated VF defects to oxygen saturation in the infero- and superotemporal retinae of patients with POAG and NTG. ${ }^{12}$ Olafsdottir et al found a significant increase in venular oxygen saturation and decreased AV difference in open-angle glaucoma subjects, ${ }^{11,13}$ suggesting reduced oxygen requirements in the atrophic tissue. However, they did not correlate with any retinal nerve fiber layer (RNFL) data or alternative VF parameters. Vandewalle et al found similar oximetry results and correlated it structurally with RNFL thickness. ${ }^{14}$ A study by Ramm et al showed similar oximetry results with earlier studies, but additionally found no difference in arterial and venous diameters between healthy controls and open-angle glaucoma subjects. ${ }^{15}$

There are questions that have not been addressed by these studies. ${ }^{10-15}$ First, the role of oxygen metabolism in PACG has not been investigated. PACG is the most obvious manifestation of pressure-dependent glaucoma; its inclusion and simultaneous comparison would yield valuable insights into the role of retinal oxygenation in the other types of glaucoma. Second, being intersubject studies, previously published results are confounded by variability in the systemic health of the patients, and thus cannot provide insight into why some patients have unilateral or asymmetrical disease, and why disease severity in some is not correlated to IOP. ${ }^{16}$

Hence, the objective of this study was to first to assess and compare retinal vessel oxygenation between the spectrum of PACG, POAG, NTG, and normal controls and second, to compare retinal oximetry between eyes with differing severity of glaucoma.

\section{Materials and methods Study design}

This prospective observational comparative study was approved by the institutional review board of the Singapore
Eye Research Institute and was conducted in adherence with the tenets of the Declaration of Helsinki. Written informed consent was obtained from each subject. Subjects aged 40 years and above and diagnosed with PACG, POAG, or NTG and normal healthy subjects were recruited from the outpatient clinics of Singapore National Eye Centre.

\section{Clinical assessment}

Thorough medical and ophthalmic history was collected, and all subjects underwent a standardized eye examination which included visual acuity, slit-lamp examination, stereoscopic evaluation of the optic disc using a 78-diopter lens, IOP measurement using Goldmann applanation tonometry, and static automated perimetry (Humphrey visual field [HVF]; Carl Zeiss Meditec AG, Jena, Germany; Swedish Interactive Threshold Algorithm 24-2 or 10-2 test pattern) with near refractive correction. A glaucomatous field defect was diagnosed and considered reliable if the glaucoma hemifield test results were graded "outside normal limits", and there was a cluster of three contiguous points at $5 \%$ level on the pattern deviation plot. Reliability criteria were as recommended by the instrument's algorithm (fixation losses, $<20 \%$; false positive and false negative, $<33 \%$ ). The VFs were scored using the Advanced Glaucoma Intervention Study (AGIS) II system. ${ }^{17}$ Comparisons with respect to interocular asymmetry of field defects between the groups were made from global indices.

Spectral-domain optical coherence tomography (Cirrus OCT; Carl Zeiss Meditec AG), using 200×200 cube Optic Disc Scan protocol, was used to measure peripapillary RNFL thickness. Only the best images obtained with a minimum signal strength $\geq 5$ were used for assessment.

\section{Retinal oximetry}

Retinal oxygen saturation measurements were taken in both eyes using a noninvasive spectrophotometric retinal oximeter (Oxymap Retinal Oximeter T1; Oxymap ehf, Reykjavik, Iceland). All fundus maps were taken after pupillary dilation with $1 \%$ tropicamide and in darkness by a single trained operator masked to the clinical diagnosis. Each subject spent 2 minutes in darkness before oximetry. Only optic disc-centered, evenly lit Oxymap retinal oximeter images were accepted. The mode of action has been well described elsewhere, and the measurement technique follows manufacturer recommendations. ${ }^{13,18,19}$ In brief, by taking two images with two different light wavelengths (570 and $600 \mathrm{~nm}$ light), the Oxymap retinal oximeter utilizes the different spectrum of light absorbance between oxyhemoglobin and 
deoxyhemoglobin to determine the oxygen saturation. The software measures vessel diameter by calculating the pixels across a cross-section segment. This is automatically converted to measurements in micrometers. ${ }^{19}$

The measurement protocol was as follows. First, a circle centered on the optic disc with radius of 20 pixels larger than the optic disc was drawn followed by a second circle 200 pixels from the first circle. Vessel measurements of the superonasal, superotemporal, inferonasal and inferotemporal arterole, and venule were taken in the area between the first and second circles (Figure 1). If the vessel trunk was $<50$ pixels in length, it was excluded and the daughter vessels were measured separately (Figure 2). Vessels with diameters $<6$ pixels in diameter or $<100$ pixels in length were excluded. Corresponding color fundus photo was viewed alongside Oxymap retinal oximeter images for comparison.

\section{Diagnostic definitions}

The three glaucoma subtypes were diagnosed according to the International Society of Geographic and Epidemiologic Ophthalmology by glaucoma fellowship-trained ophthalmologists: ${ }^{20}$

- PACG - subjects with GON with compatible reliable VF loss, presence of narrow angles (defined as $\leq 180^{\circ}$ posterior trabecular meshwork of the angle circumference seen during static gonioscopy), elevated IOP $\geq 21 \mathrm{mmHg}$, and features of trabecular obstruction by peripheral iris.

- $P O A G$ - subjects with GON, IOPs $\geq 21 \mathrm{mmHg}$, and an open, normal drainage angle with no identifiable secondary pathologic processes.

- NTG - subjects with GON and an open, normal drainage angle with no identifiable secondary pathologic processes, and IOPs $\leq 21 \mathrm{mmHg}$ with diurnal phasing.

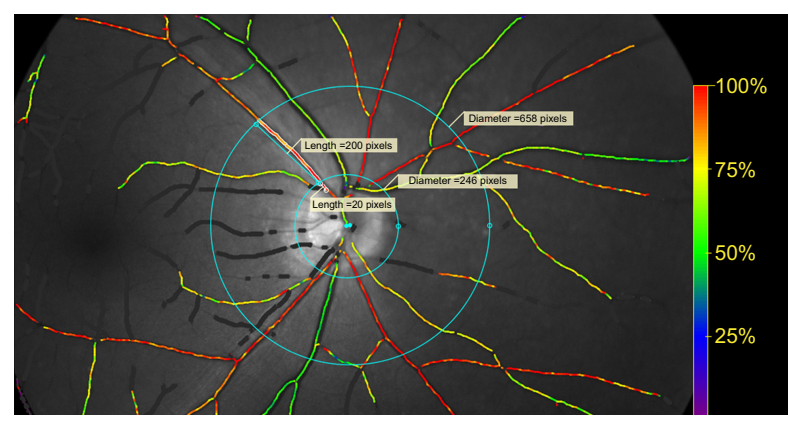

Figure I Measurement protocol.

Notes: A circle centered on the optic disc with a radius of 20 pixels was first drawn. The area within this circle was considered the no measurement zone. Next, a second circle of 200 pixels was drawn from the first circle. The area between the two circles was the measurement zone where all vessel measurements were taken.

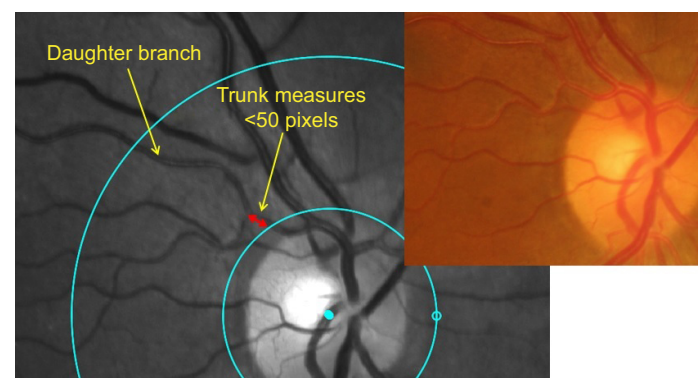

Figure 2 Example of vessel measurement.

Notes: If the vessel trunk was $<50$ pixels in length, it was excluded and the daughter vessels were measured separately. Corresponding color fundus photo was viewed alongside Oxymap retinal oximeter images for comparison.

\section{Other variables}

Blood pressure (BP), pulse rate, and finger oximetry were measured with a digital automatic monitor (Dinamap model GE Pro 100V2; GE Medical Systems, Inc., Milwaukee, WI, USA) with the patient seated after 5 minutes of rest. Finger oximetry was performed with the probe placed on the index finger of the right hand. The number of ocular hypotensive medications was also recorded with fixed combinations documented according to the number of active ingredients. Systemic carbonic anhydrase inhibitors were counted as one medication. Exclusion criteria included age-related macular degeneration, diabetic retinopathy, optic neuropathy, previous retinal laser therapy or surgery, any systemic pathology that may result in a VF defect, and pregnancy. Dry eyes and cataracts were not excluded due to their high prevalence.

The right eye of each patient was studied for baseline characteristics. The left eye was used if the retinal oximetry image quality was poor, the subject was unable to perform HVF 24-2 test pattern, or the right eye did not have glaucoma. Subjects with glaucoma in both eyes of asymmetrical severity were further analyzed for inter-eye comparison. Asymmetry was defined as an AGIS score difference of three points or more between the eyes, or if the glaucoma was so advanced in one eye that only a HVF 10-2 pattern can be performed. Subjects with bilateral HVF 10-2 test patterns were excluded in the inter-eye analysis.

\section{Statistical analysis}

Statistical analyses were performed with IBM SPSS Statistics for Windows (Version 21.0; IBM Corporation, Armonk, NY, USA). Normality of data was tested using the KolmogorovSmirnov test. As the normality assumptions were not satisfied in at least one group for a variable, nonparametric tests were used for analysis of data. For comparison among different subtypes of glaucoma, the Kruskal-Wallis test was used. Data were described in terms of mean \pm standard deviation 
for continuous variables and as frequencies and percentages for categorical variables. Wilcoxon signed-rank test was used to compare the inter-eye differences. Subanalyses were performed for the comparisons of open-angle glaucoma (POAG and NTG) versus PACG and high-pressure glaucoma (POAG and PACG) versus NTG. The differences between the two groups were examined using the Mann-Whitney $U$-test. A $P$-value $<0.05$ was considered statistically significant.

\section{Results}

One hundred and sixty-eight subjects were included in this study. One subject withdrew from the study, and another subject with ischemic optic neuropathy was excluded. Seven subjects had poor-quality images on oximetry that required exclusion. A total of 159 eyes from 159 subjects were analyzed for baseline characteristics: 39 PACG ( $n=39), 41$ POAG $(n=41)$, and 41 NTG $(n=41)$. Inter-eye differences were analyzed in 32 PACG ( $n=64), 34$ POAG (n=68), and 35 NTG $(\mathrm{n}=70)$ subjects with bilateral asymmetrical glaucoma.

The baseline characteristics are summarized in Table 1. Subjects in the PACG group were significantly older compared to normal controls. There were no differences in the duration of disease, AGIS score, and number of glaucoma medications used between the glaucoma groups. There were no differences in systolic and diastolic BP, heart rate, and pulse oximetry between all the groups. The prevalence of diabetes was found to be significantly raised in all three glaucoma groups compared to normal (PACG, $P=0.02$; POAG, $P=0.004$; NTG, $P=0.008$; Table 1).

\section{Comparison between normal and glaucomatous eyes}

Compared to normal controls, oxygen saturation was found to be significantly higher in arterioles $(P=0.048$; Table 2$)$

Table I Baseline characteristics of normal controls and glaucoma patients

\begin{tabular}{|c|c|c|c|c|c|}
\hline Characteristics & $\begin{array}{l}\text { Normal } \\
(n=38)\end{array}$ & $\begin{array}{l}\text { PACG } \\
(n=39)\end{array}$ & $\begin{array}{l}\text { POAG } \\
(n=4 I)\end{array}$ & $\begin{array}{l}\text { NTG } \\
(n=4 I)\end{array}$ & $\begin{array}{l}\text { ANOVA } \\
P \text {-value }\end{array}$ \\
\hline Age, mean $\pm S D$, years & $63.2 \pm 10.6^{\mathrm{a}}$ & $69.8 \pm 7.9^{a}$ & $66.3 \pm 11.1$ & $67.8 \pm 10.6$ & 0.038 \\
\hline Male sex, \% & 34.2 & 48.7 & 73.2 & 56.1 & NA \\
\hline Chinese race, \% (n) & $73.7(28)$ & $92.3(36)$ & $79.5(35)$ & $87.8(36)$ & NA \\
\hline Visual acuity, mean \pm SD, LogMar & $0.2 \pm 0.2$ & $0.3 \pm 0.2$ & $0.3 \pm 0.6$ & $0.2 \pm 0.2$ & 0.61 \\
\hline Duration of disease, mean $\pm S D$, years & NA & $7.1 \pm 7.6$ & $6.9 \pm 5.1$ & $5.2 \pm 4.0$ & 0.26 \\
\hline CDR, mean $\pm S D$ & $0.4 \pm 0.1^{b}$ & $0.8 \pm 0.1^{b}$ & $0.8 \pm 0.1^{b}$ & $0.8 \pm 0.1^{b}$ & $<0.001$ \\
\hline \multicolumn{6}{|l|}{$\mathrm{HVF}$, mean $\pm \mathrm{SD}$} \\
\hline MD & NA & $-17.1 \pm 9.7^{c}$ & $-13.9 \pm 8.4^{c}$ & $-8.6 \pm 6.0^{c}$ & $<0.001$ \\
\hline PSD & NA & $7.9 \pm 3.8$ & $8.2 \pm 3.7$ & $6.9 \pm 3.7$ & 0.24 \\
\hline AGIS & NA & $12.2 \pm 6.5^{d}$ & $10.0 \pm 6.1^{d}$ & $6.1 \pm 4.4^{d}$ & $<0.001$ \\
\hline $\mathrm{IOP}$, mean $\pm \mathrm{SD}, \mathrm{mmHg}$ & $15.5 \pm 2.5$ & $|4| \pm 4.2$. & $15.6 \pm 3.8$ & $13.6 \pm 3.2$ & 0.05 \\
\hline Number of glaucoma medications, mean \pm SD & NA & $1.7 \pm 0.2$ & $1.7 \pm 0.2$ & $1.8 \pm 0.1$ & 0.12 \\
\hline \multicolumn{6}{|l|}{ Glaucoma surgery, (n) } \\
\hline Trabeculectomy & NA & II & 5 & 0 & NA \\
\hline Shunt & NA & 0 & 0 & 0 & NA \\
\hline Pseudophakia, n & 9 & 23 & 23 & 15 & NA \\
\hline Systolic BP, mean $\pm \mathrm{SD}, \mathrm{mmHg}$ & $140.6 \pm 20.9$ & $150.6 \pm 21.7$ & $\mid 42.6 \pm 20.1$ & $141.9 \pm 21.2$ & 0.15 \\
\hline Diastolic BP, mean $\pm \mathrm{SD}, \mathrm{mmHg}$ & $74.5 \pm 10.4$ & $76.6 \pm 11.4$ & $76.7 \pm 11.2$ & $72.2 \pm 8.0$ & 0.16 \\
\hline Heart rate, mean $\pm S D$, bpm & $63.1 \pm 11.1$ & $63.1 \pm 11.2$ & $62.9 \pm 10.6$ & $60.5 \pm 9.9$ & 0.65 \\
\hline Pulse oximetry, mean $\pm \mathrm{SD}, \%$ & $99.0 \pm 0.9$ & $99.0 \pm 1.3$ & $98.6 \pm 1.4$ & $98.8 \pm 1.1$ & 0.43 \\
\hline \multicolumn{6}{|l|}{ Medical history, ${ }^{f} \mathrm{n}$} \\
\hline Hypertension & 13 & 20 & 24 & 23 & 0.13 \\
\hline Ischemic heart disease & 3 & 4 & 9 & 7 & 0.26 \\
\hline Hyperlipidemia & 10 & 12 & 16 & 20 & 0.17 \\
\hline Diabetes & $\mathrm{I}^{\mathrm{e}}$ & $8^{e}$ & $\mathrm{II}^{\mathrm{e}}$ & $9^{e}$ & 0.03 \\
\hline Migraine & 0 & 0 & 0 & I & 0.41 \\
\hline Anemia & 0 & 0 & I & 0 & 0.41 \\
\hline
\end{tabular}

Notes: Statistically significant $P$-values $(P<0.05)$ are shown in bold. aSignificant difference between normal controls PACG; $P=0.03$. bSignificant difference between normal controls vs PACG, POAG, NTG; $P<0.001$. 'Significant difference between NTG vs PACG, $P<0.001$; POAG, $P=0.019$. 'Significant difference between NTG vs PACG, $P<0.00$ I; POAG, $P=0.006$. eSignificant difference between normal controls vs PACG, $P=0.02$; POAG, $P=0.004 ; N T G, P=0.008$. 'Statistical analyses for categorical comparisons were performed using chi-square or Fisher's exact test appropriately. For diabetes, a further two-group comparison was done to assess the two-group differences.

Abbreviations: AGIS, Advanced Glaucoma Intervention Study; SD, standard deviation; bpm, beats per minute; BP, blood pressure; mmHg, millimeter of mercury; CDR, cup-todisc ratio; IOP, intraocular pressure; HVF, Humphrey visual field; MD, mean deviation; PSD, pattern standard deviation; PACG, primary angle-closure glaucoma; POAG, primary open-angle glaucoma; NA, not applicable; NTG, normal-tension glaucoma; LogMar, logarithm of the minimum angle of resolution; ANOVA, analysis of variance. 
Table 2 Comparison of retinal oximetry, vessel diameter, and RNFL thickness between normal controls, PACG, POAG, and NTG

\begin{tabular}{|c|c|c|c|c|c|}
\hline & $\begin{array}{l}\text { Normal }(n=38) \\
\text { mean } \pm \text { SD }\end{array}$ & $\begin{array}{l}\text { PACG }(n=39) \\
\text { mean } \pm \text { SD }\end{array}$ & $\begin{array}{l}\text { POAG }(n=4 I), \\
\text { mean } \pm \text { SD }\end{array}$ & $\begin{array}{l}\text { NTG }(n=4 I), \\
\text { mean } \pm \text { SD }\end{array}$ & $P$-value ${ }^{a}$ \\
\hline \multicolumn{6}{|c|}{ Oxygen saturation (\%) } \\
\hline Arteriole & $94.8 \pm 7.4$ & $104.7 \pm 16.4^{b}$ & $102.8 \pm 16.0$ & $99.4 \pm 13.2$ & 0.04 \\
\hline Venule & $51.9 \pm 9.9$ & $54.3 \pm 13.7$ & $53.7 \pm 13.2$ & $55.0 \pm 13.1$ & 0.13 \\
\hline AV difference & $42.8 \pm 8.8$ & $50.4 \pm 17.9$ & $49.1 \pm 16.6$ & $44.5 \pm 13.8$ & 0.19 \\
\hline \multicolumn{6}{|c|}{ Vessel diameter $(\mu \mathrm{m})$} \\
\hline Arteriole & $120.4 \pm 16.6$ & $110.4 \pm 17.5$ & $113.4 \pm 22.4$ & $116.7 \pm 15.7$ & 0.10 \\
\hline Venule & $170.8 \pm \mid 5.3$ & $152.6 \pm 19.7^{c}$ & $153.6 \pm 22.5^{c}$ & $156.5 \pm 15.3^{c}$ & $<0.00 \mathrm{I}$ \\
\hline OCT RNFL & $94.9 \pm 9.0$ & $81.4 \pm 16.3^{d}$ & $71.2 \pm 13.6^{d}$ & $79.0 \pm 14.5^{d}$ & $<0.001$ \\
\hline
\end{tabular}

Notes: Statistically significant values $(P<0.05)$ are shown in bold. ${ }^{2}$ Kruskal-Wallis test. ${ }^{b}$ Significant difference between normal controls vs $P A C G, P=0.048$. cNormal controls vs PACG, POAG, $P=0.00$ I (for both); normal controls vs NTG, $P=0.004$. ${ }^{\circ}$ Normal controls vs PACG, POAG, NTG, $P=0.00$ I (for all); PACG vs POAG, $P=0.02$.

Abbreviations: SD, standard deviation; PACG, primary angle-closure glaucoma; POAG, primary open-angle glaucoma; NTG, normal-tension glaucoma; RNFL, retinal nerve fiber layer; AV, arteriovenous; OCT, optical coherence tomography.

in PACG eyes only. There were no statistical differences in oxygen saturation of the venules $(P=0.13$; Table 2$)$ or AV difference $(P=0.19$; Table 2$)$ between PACG, POAG, NTG, and normal controls. Venular diameter was significantly reduced in all glaucoma groups compared to normal controls (PACG, $P=0.001$; POAG, $P=0.001$; NTG, $P=0.004$; Table 2). Arteriolar diameter tended to be lower in the glaucoma groups, although this was not found to be statistically significant $(P=0.10$; Table 2).

\section{Comparison between high-pressure and normal-pressure glaucoma, open-angle and closed-angle glaucoma}

All the subjects within the glaucoma groups were rearranged according to the anatomical or pressure classification and compared. There were no significant differences in the oxygen saturation in the arterioles, venules, or AV difference when high-pressure glaucoma (PACG and POAG, $\mathrm{n}=80$ ) was compared with normal-pressure glaucoma (NTG, $n=41$ ), or when open-angle glaucoma (POAG and NTG, $n=82$ ) was compared with closed-angle glaucoma group (PACG, $\mathrm{n}=39$ ). There were also no significant differences in the vessel diameters between the groups.

\section{Inter-eye comparison}

When glaucoma subjects with asymmetrical severity were compared, the worse eye in the PACG group was observed to have significantly higher arteriolar oxygen saturation (103.5 \pm 17.3 vs $97.4 \pm 9.2, P=0.03$ ), greater $\mathrm{AV}$ difference (50.9 \pm 17.9 vs $42.4 \pm 12.4, P=0.04)$, and lower arteriolar diameter $(109.7 \pm 17.3 \mu \mathrm{m}$ vs $120.7 \pm 16.8 \mu \mathrm{m}, P=0.001$; Table 3). A similar trend was observed in the POAG group, but the difference was not statistically significant. Oxygen saturation and vessel diameters between the worse and the better eyes were not significantly different in the NTG group.

\section{Discussion}

This is the first study to investigate retinal oximetry in PACG, as well as compare oximetry in patients with bilateral

Table 3 Comparison of retinal oximetry, vessel diameter, and RNFL thickness between eyes with asymmetrical severity in PACG, POAG, and NTGa

\begin{tabular}{|c|c|c|c|c|c|c|c|c|c|}
\hline & \multicolumn{3}{|c|}{ PACG $(n=64)$} & \multicolumn{3}{|c|}{ POAG $(n=68)$} & \multicolumn{3}{|l|}{ NTG $(n=70)$} \\
\hline & $\begin{array}{l}\text { Worse eye, } \\
\text { mean } \pm \text { SD }\end{array}$ & $\begin{array}{l}\text { Better eye, } \\
\text { mean } \pm \text { SD }\end{array}$ & $P$-value & $\begin{array}{l}\text { Worse eye, } \\
\text { mean } \pm \text { SD }\end{array}$ & $\begin{array}{l}\text { Better eye, } \\
\text { mean } \pm \text { SD }\end{array}$ & $P$-value & $\begin{array}{l}\text { Worse eye, } \\
\text { mean } \pm \text { SD }\end{array}$ & $\begin{array}{l}\text { Better eye, } \\
\text { mean } \pm \text { SD }\end{array}$ & $P$-value \\
\hline \multicolumn{10}{|c|}{ Oxygen saturation (\%) } \\
\hline Arteriole & $103.5 \pm 17.3$ & $97.4 \pm 9.2$ & 0.03 & $104.9 \pm 16.6$ & $99.7 \pm 13.9$ & 0.14 & $99.3 \pm 15.7$ & $98.7 \pm 12.0$ & 0.96 \\
\hline Venule & $53.7 \pm 14.2$ & $53.6 \pm 13.6$ & 0.75 & $55.1 \pm 13.2$ & $53.0 \pm 12.4$ & 0.12 & $55.5 \pm 13.9$ & $54.7 \pm 10.5$ & 0.52 \\
\hline AV difference & $50.9 \pm 17.9$ & $42.4 \pm I 2.4$ & 0.04 & $49.8 \pm 16.9$ & $46.7 \pm 13.9$ & 0.50 & $44.3 \pm 16.3$ & $43.9 \pm 12.3$ & 0.82 \\
\hline \multicolumn{10}{|c|}{ Vessel diameter $(\mu \mathrm{m})$} \\
\hline Arteriole & $109.7 \pm 17.3$ & $|20.7 \pm| 6.8$ & 0.001 & $112.5 \pm 23.1$ & $115.9 \pm 23.7$ & 0.32 & $|14.4 \pm| 4 . \mid$ & $117.8 \pm 19.7$ & 0.22 \\
\hline Venule & $152.8 \pm 19.3$ & $156.3 \pm 19.4$ & 0.34 & $155.4 \pm 22.0$ & $|56.7 \pm 2| .2$ & 0.59 & $158.5 \pm \mid 5.3$ & $156.7 \pm 18.6$ & 0.61 \\
\hline OCT RNFL & $64.1 \pm 11.7$ & $84.0 \pm I 5.4$ & $<\mathbf{0 . 0 0 1}$ & $62.1 \pm 10.3$ & $73.4 \pm 13.6$ & $<0.001$ & $71.8 \pm 9.6$ & $80.8 \pm I 4.5$ & $<0.001$ \\
\hline
\end{tabular}

Notes: aWilcoxon signed-rank test. Statistically significant values $(P<0.05)$ are shown in bold.

Abbreviations: SD, standard deviation; PACG, primary angle-closure glaucoma; POAG, primary open-angle glaucoma; NTG, normal-tension glaucoma; RNFL, retinal nerve fiber layer; AV, arteriovenous; OCT, optical coherence tomography. 
glaucoma of asymmetrical severity. Our results showed arteriolar oxygen saturation was higher in PACG eyes but not in POAG and NTG eyes compared to normal controls. Venular oxygen saturation and AV difference were similar between PACG, POAG, NTG, and normal controls. This is contrary to other studies published on the matter of retinal oximetry and open-angle glaucoma, ${ }^{10-14}$ where an increase in venular oxygen saturation and reduction in AV difference were observed and thought to reflect reduced oxygen demand due to tissue loss. Reliable and reproducible measurement of retinal vessel oximetry using the Oxymap Retinal Oximeter T1 has been shown in another study with similar Singaporean study population as ours. ${ }^{21}$ The difference observed in our study could be related to the increased pigmentation in the South Asian population on oximetric measurements as melanin is known to alter the reflectance spectra from the fundus. ${ }^{22}$

When comparing between eyes of asymmetrical severity, we observed that arteriolar oxygen saturation and AV difference were higher and arteriolar diameter was lower in the worse eye of the PACG group. Oximetric parameters between eyes of asymmetric severity in POAG showed a similar trend, although it was not found to be statistically significant, while results from the NTG group were comparable. While lower retinal arteriolar diameter can be explained by vasoconstriction in response to higher oxygen saturation, it is unclear why there is a relative "hyperoxic" state in PACG eyes and in eyes with more advanced disease. One hypothesis would be an increased tissue metabolic demand in the compromised state in PACG, rather than a reduction as previously proposed in other studies, resulting in higher arteriolar oxygen saturation and AV difference.

The Oxymap analyzer software has been shown to measure vessel diameter in a consistent and repeatable manner and hence will allow detection of clinically important differences in vessel diameter. ${ }^{23}$ In our study, we found changes in the vessel caliber between normal controls and glaucoma subjects. Venular diameter was significantly reduced in PACG, POAG, and NTG eyes compared to normal controls. Arteriolar diameter was not found to be different between the glaucoma groups and normal controls. When divided according to severity, arteriolar diameter was significantly lower in PACG eyes with more severe disease. There were no significant differences in the arteriolar diameter between the eyes in POAG and NTG groups. As the RNFL thickness difference between the worse and the better eyes in PACG was twice that of POAG and NTG in our study group, it is possible that the difference in severity in POAG and NTG was not substantial enough to show a significant difference in vessel caliber. There was no difference in any of the parameters when the glaucoma subjects were classified according to the presenting IOP or anatomical structure.
Retinal vessel narrowing in glaucoma has been reported elsewhere. ${ }^{24,25}$ Amerasinghe et al showed that even after factoring the effects of BP, smoking, age, IOP, and other risk factors, subjects with narrower retinal vessels were more likely to have glaucoma and larger vertical cup-to-disc ratio. Furthermore, there was no association between retinal vascular caliber and IOP. ${ }^{26}$ In that study, which included a Singaporean population similar to our study, the authors were not able to show any association with vessel caliber in PACG due to the insufficient sample size. ${ }^{25}$

While the exact effect of reduction on vessel caliber in glaucoma is yet to be proven, it could be related to Poiseuille's formula and its role in blood volume flow rate to the optic nerve head and retina. In Poiseuille's formula, providing the pressure-gradient remains constant, the volume flow varies to the fourth power of the radius of the tube. That is to say a small decrease in vessel radius can significantly decrease the volume flow rate. This has been shown to occur in human retinal blood flow. ${ }^{26}$ Reduction in vessel caliber also leads to increased vascular resistance. Both factors play a role in the distal provision of oxygen to the retina and optic nerve head.

In our subject cohort, we produced arterial oxygen saturation values that exceeded $100 \%$. This has been known to occur $^{19}$ and could be due to calibration error and not accounting for the variations in pigmentation of the retina in different ethnic groups. ${ }^{22}$ However, this is unlikely to influence results as the relationship between the optical density ratio and the eventual calculated oxygen saturation value is linear.

There were some limitations in our study. Our study cohort observed patients who had received treatment for glaucoma. We did not know the effect of IOP on pressure gradient and vessel caliber at the onset of the disease. We also did not know the perfusion pressure and could not assess autoregulatory mechanisms of the retinal vessels. Furthermore, it is possible that poor regulation of blood flow, which has been postulated to occur in glaucoma, can result in intermittent changes in oxygenation that may not have been captured in our study. As our study included only crosssectional data, we do not have the temporal information of these associations to determine whether narrowing of retinal vessels reflect ischemia resulting in damage to the optic nerve or are a consequence of tissue atrophy in GON. Our study also included a small number of diabetic patients of varying disease duration which could confound our results. With these limitations, until automated measurements are available, at its present form, poor user interface and laborious measurements relegate the retinal oximeter to being a research tool.

The strengths of our study are threefold. We performed 1) quantitative assessment of retinal oximetry in PACG, 
2) inter-eye comparison between eyes of asymmetrical severity control for systemic variability between subjects, and 3) simultaneous assessments of retinal oximetry and vessel caliber in these subjects, which allow for more detailed assessment of the vascular component in glaucoma.

In conclusion, we demonstrated that there are differences in oxygen saturation in retinal vessels in PACG compared to normal controls, POAG, and NTG eyes. Vessel caliber is reduced in glaucoma compared to normal controls and appears to be inversely related to severity of disease. Further studies including ocular hypertensives, treatment-naïve glaucoma subjects, and longitudinal follow-up are needed to evaluate the influence of retinal vessel diameter in glaucoma.

\section{Acknowledgments}

The authors would like to acknowledge the contributions from the Singapore National Eye Centre Medical Illustration team. This study received funding from the SingHealth Foundation Research Grant SHF/FG454P/2011.

\section{Disclosure}

The authors report no conflicts of interest in this work.

\section{References}

1. Boeckaert J, Vandewalle E, Stalmans I. Oximetry: recent insights into retinal vasopathies and glaucoma. Bull Soc Belge Ophtalmol. 2012; (319):75-83.

2. Sigal IA, Ethier CR. Biomechanics of the optic nerve head. Exp Eye Res. 2009;88:799-807.

3. Gazzard G, Foster PJ, Viswanathan AC, et al. The severity and spatial distribution of visual field defects in primary glaucoma: a comparison of primary open-angle glaucoma and primary angle-closure glaucoma. Arch Ophthalmol. 2002;120:1636-1643.

4. Mozaffarieh M, Grieshaber MC, Flammer J. Oxygen and blood flow: players in the pathogenesis of glaucoma. Mol Vis. 2008;14:224-233.

5. Caprioli J. Neuroprotection of the optic nerve in glaucoma. Acta Ophthalmol Scand. 1997;75:364-367.

6. Morgan J, Caprioli J, Koseki Y. Nitric oxide mediates excitotoxic and anoxic damage in rat retinal ganglion cells cocultured with astroglia. Arch Ophthalmol. 1999;117:1524-1529.

7. Fontana L, Poinoosawmy D, Bunce CV, O'Brien C, Hitchings RA. Pulsatile ocular blood flow investigation in asymmetric normal tension glaucoma and normal subjects. Br J Ophthalmol. 1998;82(7):731-736.

8. Arend O, Remky A, Cantor LB, Harris A. Altitudinal visual field asymmetry is coupled with altered retinal circulation in patients with normal pressure glaucoma. Br J Ophthalmol. 2000;84(9):1008-1012.

Clinical Ophthalmology

\section{Publish your work in this journal}

Clinical Ophthalmology is an international, peer-reviewed journal covering all subspecialties within ophthalmology. Key topics include: Optometry; Visual science; Pharmacology and drug therapy in eye diseases; Basic Sciences; Primary and Secondary eye care; Patient Safety and Quality of Care Improvements. This journal is indexed on
9. Butt Z, O’Brien C, McKillop G, Aspinall P, Allan P. Color Doppler imaging in untreated high- and normal-pressure open-angle glaucoma. Invest Ophthalmol Vis Sci. 1997;38(3):690-696.

10. Michelson G, Scibor M. Intravascular oxygen saturation in retinal vessels in normal subjects and open-angle glaucoma subject. Acta Ophthalmol Scand. 2006;84(3):289-295.

11. Olafsdottir OB, Vandewalle E, Pinto LA, et al. Retinal oxygen metabolism in healthy subjects and glaucoma patients. Br J Ophthalmol. 2014; 98(3):329-333.

12. Ito M, Murayama K, Deguchi T, et al. Oxygen saturation levels in the juxta-papillary retina in eyes with glaucoma. Exp Eye Res. 2008;86(3): 512-518.

13. Olafsdottir OB, Hardarson SH, Gottfredsdottir MS, Harris A, Stefánsson E. Retinal oximetry in primary open-angle glaucoma. Invest Ophthalmol Vis Sci. 2011;52(9):6409-6413.

14. Vandewalle E, Abegão Pinto L, Olafsdottir OB, et al. Oximetry in glaucoma: correlation of metabolic change with structural and functional damage. Acta Ophthalmol. 2014;92(2):105-110.

15. Ramm L, Jentsch S, Peters S, Augsten R, Hammer M. Investigation of blood flow regulation and oxygen saturation of the retinal vessels in primary open-angle glaucoma. Graefes Arch Clin Exp Ophthalmol. 2014; 252(11):1803-1810.

16. Poinoosawmy D, Fontana L, Wu JX, Bunce CV, Hitchings RA. Frequency of asymmetric visual field defects in normal tension and high tension glaucoma. Ophthalmology. 1998;105(6):988-991.

17. The Advanced Glaucoma Intervention Study Investigators. Advanced Glaucoma Intervention Study 2. Visual field test scoring and reliability. Ophthalmology. 1994;101(8):1445-1455.

18. Hardarson SH, Harris A, Karlsson RA, et al. Automatic retinal oximetry. Invest Ophthalmol Vis Sci. 2006;47(11):5011-5016.

19. Hardarson SH. Retinal oximetry. Acta Ophthalmol. 2013;91(5): 489-490.

20. Foster PJ, Buhrmann R, Quigley HA, Johnson GJ. The definition and classification of glaucoma in prevalence surveys. Br J Ophthalmol. 2002;86(2):238-242.

21. Yip W, Siantar R, Perera SA, et al. Reliability and determinants of retinal vessel oximetry measurements in healthy eyes. Invest Ophthalmol Vis Sci. 2014;55(11):7104-7110.

22. Delori FC, Pflibsen KP. Spectral reflectance of the human ocular fundus. Appl Opt. 1989;28(6):1061-1077.

23. Blondal R, Sturludottir MK, Hardarson SH, Halldorsson GH, Stefánsson E. Reliability of vessel diameter measurements with a retinal oximeter. Graefes Arch Clin Exp Ophthalmol. 2011;249(9):1311-1317.

24. Mitchell P, Leung H, Wang JJ, et al. Retinal vessel diameter and open-angle glaucoma: the Blue Mountains Eye Study. Ophthalmology. 2005;112(2):245-250.

25. Amerasinghe N, Aung T, Cheung N, et al. Evidence of retinal vascular narrowing in glaucomatous eyes in an Asian population. Invest Ophthalmol Vis Sci. 2008;49(12):5397-5402.

26. Feke GT, Tagawa H, Deupree DM, Goger DG, Sebag J, Weiter JJ. Blood flow in the normal human retina. Invest Ophthalmol Vis Sci. 1989; 30(1):58-65.

PubMed Central and CAS, and is the official journal of The Society of Clinical Ophthalmology (SCO). The manuscript management system is completely online and includes a very quick and fair peer-review system, which is all easy to use. Visit http://www.dovepress.com/ testimonials.php to read real quotes from published authors. 